\title{
IBD: reproductive health, pregnancy and lactation
}

\author{
Philip Hendy, ${ }^{1}$ Georgina Chadwick, ${ }^{1}$ Ailsa Hart ${ }^{2}$
}

${ }^{1}$ Gastroenterology SPRs, St Mark's Hospital, Harrow, UK ${ }^{2}$ Gastroenterology, St Mark's Hospital, Harrow, UK

\section{Correspondence to} Dr Philip Hendy, IBD Unit, St Mark's Hospital, Watford Rd, Harrow, HA1 3UJ, UK; philiphendy14@gmail.com

Received 2 January 2014 Revised 26 March 2014 Accepted 1 April 2014 Published Online First 15 April 2014

\section{CrossMark}

To cite: Hendy $P$,

Chadwick G, Hart A. Frontline Gastroenterology 2015;6:

38-43.

\begin{abstract}
The peak incidence of inflammatory bowel diseases (IBD) occurs during the child-bearing years, and so questions about fertility, pregnancy and breast feeding are often asked by patients. It is known that patients with IBD receive twice as much information about pregnancy-related issues from gastroenterologists as from any other source (including the internet). Therefore, the role of the gastroenterologist in correctly educating patients to avoid misconceptions is paramount, and this should be done proactively prior to planning pregnancy so that the patient's health and medications can be optimised. A clear understanding of medication safety during pregnancy and lactation will improve clinical management.
\end{abstract}

\section{INTRODUCTION}

Inflammatory bowel diseases (IBD) affects over 200000 patients in the UK. The majority of sufferers develop the disease during their reproductive years, and so the management of IBD during pregnancy is a common situation encountered by clinicians.

Competency 3b (box 9) of the 2010 gastroenterology curriculum stipulates that trainees should understand the effect of IBD and its treatment on reproductive health, pregnancy and lactation. This article reviews the effects of IBD on fertility and pregnancy outcomes, and the safety of IBD medications during pregnancy and breast feeding (see box 1).

\section{FERTILITY AND IBD}

Fertility is defined as the rate of reproduction, or number of live births recorded, for an individual or a population. Fecundity, meanwhile, relates to the potential for an individual to reproduce. A woman may have high fecundity (biological ability to conceive) and yet have low fertility, for example, through the intentional use of contraception or abstinence. Overall, patients with IBD have similar fertility rates to the general population, ${ }^{1}{ }^{2}$ but particular subgroups of patients may be affected by reduced fertility and fecundity.

\section{ULCERATIVE COLITIS}

Patients with ulcerative colitis (UC) who are surgery-naive, do not seem to have reduced fertility rates. ${ }^{3}$ It is generally accepted that previous abdominal or pelvic, in particular pouch, surgery reduces fertility to rates of up to $48 \% .{ }^{4}$ Newer studies suggest that laparoscopic surgery preserves fertility when compared with open procedures, with fertility rates after laparoscopic pouch formation approximating those in the general population. ${ }^{56}$

\section{CROHN'S DISEASE}

Patients with Crohn's disease (CD) have, overall, reduced fertility when compared with healthy controls, ${ }^{7}$ and have relatively fewer children after the diagnosis of CD is made, as compared with before diagnosis. Patients with quiescent disease have normal fertility, ${ }^{8}$ whereas those with active disease or with previous abdominal or pelvic surgery have reduced fertility. A recent systematic review of fertility in surgery-naive patients with $\mathrm{CD}$ showed that the most important factor was voluntary childlessness, ${ }^{7}$ implying that reduced fecundity may not be as significant as previously thought. The reasons for voluntary childlessness in CD are likely to be multifactorial, and probably include concerns about reduced fertility, anxieties around hereditability, worries about the effects of pregnancy on the disease, and general fears about medications in pregnancy and breast feeding. Many of these concerns are not grounded in evidence. Aside from voluntary childlessness, CD 
Box 1 Gastroenterology curriculum 2010 Competency 3b (box 9)

Understanding the effect of IBD and its treatment on reproductive health, pregnancy and lactation

- Knows the effect of active IBD, drug therapy and surgery on fecundity and pregnancy, specifically issues relating to immune suppressants, biological therapy and surgery.

- Knows the effect of IBD and its treatment on breast feeding.

- Knows the effects of the disease and its treatment on the chances of conception for men with IBD.

may reduce fertility through pelvic inflammation and through reduced frequency of sexual intercourse (due to dyspareunia, altered body image and diarrhoea).

\section{Hereditability}

If one parent has IBD, the offspring has a 10-fold increase in the risk of developing IBD $^{9}$ (around 7\%), and the risk is even higher when both parents have $\mathrm{IBD}^{10}$ (up to $35 \%$ ). CD has greater hereditability than UC. The fear of passing the disease onto offspring may contribute to the high levels of voluntary childlessness.

\section{Drugs and fertility}

The main drug classes used to treat IBD do not seem to reduce fertility in women, but methotrexate and sulfasalazine are known to reduce sperm production and motility in men (methotrexate is also highly teratogenic and should be avoided in any case in males and females considering starting a family).

Table 1 Based on ECCO consensus guidelines; safety of IBD drugs in pregnancy ${ }^{17}$

\begin{tabular}{lll}
\hline Safe & $\begin{array}{l}\text { Probably } \\
\text { safe }\end{array}$ & Contraindicated \\
\hline $\begin{array}{l}\text { Oral 5-aminosalicylates } \\
\text { Topical 5-aminosalicylates } \\
\text { Sulfasalazine }\end{array}$ & $\begin{array}{l}\text { Infliximab } \\
\text { Adalimumab } \\
\text { Certolizumab }\end{array}$ & $\begin{array}{l}\text { Methotrexate } \\
\text { Thalidomide } \\
\text { 6-Thioguanine } \\
\text { (no data) }\end{array}$ \\
$\begin{array}{l}\text { Corticosteroids } \\
\text { Azathioprine } \\
\text { 6-mercaptopurine } \\
\text { Oral 5-aminosalicylates }\end{array}$ & $\begin{array}{l}\text { Cyclosporin } \\
\text { Tacrolimus }\end{array}$ & \\
& $\begin{array}{l}\text { Metronidazole } \\
\text { Ciprofloxacin }\end{array}$ & \\
\hline
\end{tabular}

ECCO, European Crohn's and Colitis Organisation; IBD, Inflammatory bowel diseases.

\section{PREGNANCY AND IBD}

Effect of pregnancy on IBD

The most important factor affecting disease activity during pregnancy is the degree of activity at the time of conception. ${ }^{11}{ }^{12}$ About a third of patients with inactive UC at the time of conception will have a flare of their disease during the following 12 months; similar to non-pregnant individuals with UC. For patients with active UC at the time of conception, around half will deteriorate during pregnancy, a quarter will improve and a quarter will remain stable.

Similar findings are seen in patients with CD. With inactive disease at conception, only a quarter of patients will experience a flare during pregnancy. Active disease at conception is associated with the rule of thirds with regards to ongoing disease activity; a third of patients will experience a deterioration, a third will remain unchanged, and a third will improve.

\section{Effect of IBD on pregnancy outcomes}

The impact of IBD on pregnancy outcomes correlates with the level of disease activity at the time of conception. Overall, most women with inactive IBD experience an uneventful pregnancy, ${ }^{13}$ with studies suggesting that women with inactive disease at the time of conception have similar risks of miscarriage, and adverse perinatal outcomes as the general population. ${ }^{12} 1415$ However, active IBD does appear to be associated with higher preterm birth rates, low birth weights (LBW) and higher caesarean section rates, and this risk may be higher in CD than in UC. ${ }^{1}$ Active disease does not appear to affect the risk of congenital defects or stillbirth, however. ${ }^{16}$ While results from some studies are conflicting, it does appear that women with active disease at the time of conception are more likely to be affected by adverse pregnancy outcomes. It is therefore important to encourage women to aim for disease remission prior to conception, and to ensure that patients are closely monitored and appropriately treated during pregnancy.

\section{Drugs and pregnancy}

Medication is a major area of concern for patients who are pregnant or considering becoming pregnant, and discussions regarding medical management must be on an individual basis to ensure awareness of risks and benefits. Most of the medications used to treat IBD are safe in pregnancy (with the important exceptions of methotrexate, mycophenalate and thalidomide (see table 1)), but the strength of evidence to substantiate this varies from drug to drug, and hence all medication decisions must be made after detailed discussion with the patient. Maternal wellness must be the over-riding aim of management during pregnancy, for the mother and fetus, because flares of IBD and surgery for IBD predict worse fetal outcome. In one large population-based study, IBD medication was 
discontinued by sixteen percent of pregnant females. The two most frequent reasons for discontinuation were personal choice and medical advice. These figures represent an opportunity to improve patient care through the education of pregnant patients and medical professionals.

\section{Sulfasalazine}

As sulfasalazine is a folic acid antagonist, high-dose folic acid ( $2 \mathrm{mg} /$ day) should be commenced around the time of conception to reduce the risk of fetal neural tube defects. Although sulfasalazine crosses the placenta, there is little evidence that it may harm the fetus.

As mentioned above, sulfasalazine is known to decrease sperm motility and numbers, and may theoretically reduce male fertility (although this has not been shown).

5-aminosalicylic acid

Mesalazine is poorly absorbed from the gastrointestinal tract and does not cross the placenta in significant quantities, and so maintenance doses $(<3 \mathrm{~g}$ /day) are considered safe in pregnancy. ${ }^{18}{ }^{19}$ Higher doses may be associated with infant interstitial nephritis ${ }^{20}$ and should be avoided.

\section{Corticosteroids}

Steroids do cross the placenta (indeed corticosteroid is administered to the mother in cases of premature labour to expedite fetal lung maturation) but are not teratogenic, and have been shown to be a reasonable option in pregnancy (although some retrospective data suggest an increased risk of cleft palate, prospective studies revealed no such association). ${ }^{21}$ During pregnancy, steroids may be used to recapture remission in moderate to severe disease activity. Blood sugar should be monitored closely in those at high risk of gestational diabetes.

\section{Thiopurines}

Azathioprine has been used during pregnancy in transplant and rheumatology patients for some time, and there is now increasing evidence that it is also a reasonable option for patients with IBD during pregnancy particularly if it enables good disease control. $^{2223}$ A recent meta-analysis revealed that thiopurines are not associated with LBW or congenital abnormalities, but may be associated with preterm delivery. $^{23} 24$

Maternal thiopurine metabolism is altered during pregnancy, with decreased 6-thioguanine (TGN) and raised 6-methylmercaptopurine (MMP) levels, but this does not seem to impact on maternal health. ${ }^{25}$ The thiopurine metabolite 6-TGN does cross the placenta, but again there are few data to suggest poor fetal outcome. While it is a reasonable option to continue thiopurine therapy when a patient becomes pregnant, after careful discussion with the patient, it is wise to avoid starting it during pregnancy because the potential adverse drug reactions occur most frequently on commencement of medication, rather than when it is in steady state. Offspring of men who take thiopurines do not seem to suffer adverse outcomes, but the data are mixed. ${ }^{26} 27$ A 485 patient retrospective cohort study comparing conception and pregnancy outcomes of thiopurine versus non-thiopurine-treated IBD patients did not find a worse outcome in the treatment group. ${ }^{28}$

\section{Cyclosporine}

The calcineurin inhibitor, cyclosporine, decreases lymphocyte synthesis of inflammatory cytokines by reducing activation of cytokine transcription factors. It has been used extensively for renal transplant patients, including during pregnancy. ${ }^{29}$ Although concerns surround the use of such a potent drug, its use may be justified to avoid colectomy for severe, steroid-resistant flares of UC during pregnancy (where fetal mortality may be up to $60 \%{ }^{13}$ ). Meta-analysis has shown that there is no statistically significant increased risk to the fetus, ${ }^{30}$ and the Getaid Group concluded that cyclosporine for steroid-refractory UC during pregnancy can be considered safe and effective.

\section{Methotrexate, mycophenalate and thalidomide}

These drugs are highly teratogenic and confer disastrous fetal outcome. Strict adherence to contraception must be practiced while a patient (male or female) is taking methotrexate, mycophenalate or thalidomide, and for 6 months after cessation of the drug.

\section{Allopurinol}

The antimetabolite, allopurinol, is increasingly used in IBD to help reduce the shunt towards MMP during thiopurine metabolism. There is little experience of allopurinol in pregnancy because its primary indication, gout, tends to present in an older population. There is some evidence to suggest that allopurinol may be a teratogen, and it should be used with care in the first trimester. ${ }^{31}$ Allopurinol is occasionally administered to pregnant women at or near term to reduce the neonatal neurological sequelae of free radical oxygen molecules produced by hypoxia and other inflammatory states. ${ }^{32}$ A personalised discussion of the benefits and risks of the medication must be had with any patient who is pregnant or considering starting a family.

\section{Anti-tumour necrosis factor}

There is increasing evidence that anti-tumour necrosis factor (anti-TNF) drugs are reasonable options in pregnancy for patients with IBD. ${ }^{33}$ The largest study is from the Pregnancy in Inflammatory Bowel Disease and Neonatal Outcome (PIANO) registry which is prospectively following more than 1000 women with IBD during the course of their pregnancy and beyond. ${ }^{35}$ Data from this registry show that there 
is no increase in 'any pregnancy complication' (including: small-for-age fetus, congenital abnormality, preterm birth, caesarean section, neonatal intensive care stay) as a result of anti-TNF therapy. Infant height, weight and developmental milestones at 4, 9 and 12 months of age were also not affected by maternal anti-TNF status. There was, however, a significant increase in infant infection rates at 12 months in those whose mother took anti-TNF drugs, and long-term data are awaited.

With infliximab and adalimumab, anti-TNF antibodies do not readily cross the placenta in the first trimester, but do cross quite freely during the third trimester of pregnancy, therefore, it is generally advised that medication is withheld during the third trimester, if possible, to prevent neonatal immunosuppression. There are case reports of neonatal death following live vaccination after exposure to anti-TNF in utero. ${ }^{36}$ For this reason, live vaccinations (eg, Bacillus Calmette-Guérin (BCG), measles mumps and rubella (MMR) and oral polio and diptheria vaccines) should not be given until 6 months postpartum, unless anti-TNF levels have been checked and are absent from the baby's blood. Certolizumab only crosses the placenta in low levels at any point during pregnancy, and this may be a safer option, but there are no data to support this. The combination of biological therapy and immunomodulators also seems to be a reasonable option in pregnancy. ${ }^{22}$ In men taking anti-TNFs it is not known if the fetus may be affected by antibodies in semen, therefore, barrier contraception should be used during pregnancy.

\section{Antibiotics}

Short courses of low-dose oral metronidazole may be beneficial in treating pouchitis and perianal $C D$, and are thought to be low risk in pregnant patients. Ciprofloxacin may be considered for the same indications, but the BNF advises against this. Animal studies have shown a risk of degeneration of developing cartilage following quinolone exposure, but a meta-analysis of pregnancy outcomes did not highlight any adverse effect. ${ }^{37}$

Table 2 Based on the ECCO consensus guidelines on medication safety during breast feeding ${ }^{17}$

\begin{tabular}{llll}
\hline Safe & Probably safe & Unknown & Not safe \\
\hline Mesalazine & Infliximab & Metronidazole & Methotrexate \\
Sulfasalazine & Adalimumab & Ciprofloxacin & $\begin{array}{l}\text { Cyclosporine } \\
\text { Corticosteroids }\end{array}$ \\
& Certolizumab & & Thalidomide \\
& Azathioprine & & \\
& 6-Mercaptopurine & & \\
& Tacrolimus & & \\
& Budesonide & &
\end{tabular}

ECCO, European Crohn's and Colitis Organisation.

\section{Nutritional supplements in IBD}

As with all pregnant women, patients with IBD should be advised to take folic acid supplements in the first trimester, but doses should be increased if the patient is taking sulfasalazine or if they have CD affecting the small intestine, or have had previous small bowel surgery, as this may affect drug absorption. Patients taking oral steroids should also take supplements of calcium and vitamin D. Vitamin B12 deficiency as a result of previous small bowel surgery or disease should be corrected with supplementation.

\section{Surgery and pregnancy}

The indications for emergency surgery for IBD during pregnancy are the same as for non-pregnant patients. Toxic dilatation of the colon, intestinal perforation, septic collection and complete obstruction secondary to stricture formation all require prompt surgical management.

The IBD-specific indication for caesarean section is active perianal disease, with the aim of preserving anal sphincter function. Patients with an ileal pouch anal anastamosis may also be counselled regarding a caesarean section to try to preserve faecal continence.

\section{Radiology, endoscopy and pregnancy}

Flexible sigmoidoscopy, with or without preparation, is considered to be relatively safe during pregnancy, and warranted if there is a strong indication. ${ }^{38}$ Colonoscopy is subject to greater risks and should only be performed if deemed absolutely necessary. Data suggest that colonoscopy should be performed in the second trimester, and in hospital, by a skilled endoscopist in coordination with the obstetrician. The risks of sedation must also be considered.

As with non-IBD pregnancies, ionising radiation should be avoided. There are inadequate safety data regarding the use of MRI during pregnancy for firm conclusions to be drawn. There is a theoretical risk of fetal congenital abnormalities if MRI is performed during the first trimester, but again, data are inadequate to form a consensus opinion. If the clinical picture mandates an MRI scan, then it should be performed following a detailed discussion with the patient about risks and benefits. ${ }^{39}$

\section{BREAST FEEDING AND IBD}

Women with IBD are as likely as the general population to breast feed, and breast feeding is not associated with increased risk of disease exacerbation. ${ }^{40}$ Breast feeding may be protective against IBD for the infant, but the data are not conclusive. ${ }^{41}$

Below is a table (Table 2) summarising the ECCO consensus guidelines on medication safety and breast feeding (content modified). As with medication and pregnancy, medication during breast feeding should be discussed in depth with the patient. The 5-ASAs have been used for years, and there is good evidence for 
their safety during breast feeding, with minimal 5-ASA levels found in breast milk. ${ }^{42}{ }^{43}$ There have been case reports of infant diarrhoea with maternal 5-ASA proposed as the likely cause. ${ }^{43}$ The BNF recommends monitoring the neonate for diarrhoea. Corticosteroids pass into breast milk in small quantities only, and are considered reasonable, but ECCO suggests a $4 \mathrm{~h}$ delay between medicating and breast feeding, if possible. ${ }^{17}$ There are few data regarding budesonide and breast feeding, but given its poor absorption it should be considered as a reasonable option. Thiopurines are excreted into breast milk in small volumes, and are regarded as safe. ${ }^{44}$ Prospective data from the PIANO registry have shown anti-TNFs to be reasonable during breast feeding, but as yet, there are no long-term data. ${ }^{35}{ }^{45}$ Due to lack of data, it is advised that metronidazole and ciprofloxacin are avoided during breast feeding. The deleterious effects of methotrexate, cyclosporine and thalidomide through breast feeding are well documented, and these medications are contraindicated.

\section{CONCLUSION}

The topic of pregnancy and IBD is important and complex, and best managed by specialists from the fields of gastroenterology and obstetrics. In most cases, IBD does not affect fecundity, but patients with IBD have higher than general population levels of voluntary childlessness, and this is something which should be addressed. There are clear guidelines about the safety of medications during pregnancy and the puerperium, and frank discussion of these with the patient is key to improving medication compliance and disease control. Patient education about pregnancy in the context of IBD prior to conception is vital to ensure disease and medication optimisation.

Contributors $\mathrm{PH}$ and $\mathrm{GC}$ wrote the article; $\mathrm{AH}$ reviewed and edited the manuscript.

\section{Competing interests None.}

Provenance and peer review Not commissioned; externally peer reviewed.

\section{REFERENCES}

1 Mañosa M, Navarro-Llavat M, Marín L, et al. Fecundity, pregnancy outcomes, and breastfeeding in patients with inflammatory bowel disease: a large cohort survey. Scand J Gastroenterol 2013;48:427-32.

2 Hudson M, Flett G, Sinclair TS, et al. Fertility and pregnancy in inflammatory bowel disease. Int J Gynaecol Obstet 1997;58:229-37.

3 Willoughby CP, Truelove SC. Ulcerative colitis and pregnancy. Gut 1980;21:469-74.

4 Waljee A, Waljee J, Morris AM, et al. Threefold increased risk of infertility: a meta-analysis of infertility after ileal pouch anal anastomosis in ulcerative colitis. Gut 2006;55:1575-80.

5 Beyer-Berjot L, Maggiori L, Birnbaum D, et al. A total laparoscopic approach reduces the infertility rate after ileal pouch-anal anastomosis: a 2-center study. Ann Surg 2013;258:275-82.

6 Bartels SAL, D’Hoore A, Cuesta MA, et al. Significantly increased pregnancy rates after laparoscopic restorative proctocolectomy: a cross-sectional study. Ann Surg 2012;256:1045-8.

7 Tavernier N, Fumery M, Peyrin-Biroulet L, et al. Systematic review: fertility in non-surgically treated inflammatory bowel disease. Aliment Pharmacol Ther 2013;38:847-53.

8 Baird DD, Narendranathan M, Sandler RS. Increased risk of preterm birth for women with inflammatory bowel disease. J Gastroenterol 1990;99:987-94.

9 Orholm M, Munkholm P, Langholz E, et al. Familial occurrence of inflammatory bowel disease. $\mathrm{N} \mathrm{Engl} \mathrm{J} \mathrm{Med}$ 1991;324:84-8.

10 Laharie D, Debeugny S, Peeters M, et al. Inflammatory bowel disease in spouses and their offspring. J Gastroenterol 2001;120:816-9.

11 Beaulieu DB, Kane S. Inflammatory bowel disease in pregnancy. World J Gastroenterol 2011;17:2696-701.

12 Miller JP. Inflammatory bowel disease in pregnancy: a review. J R Soc Med 1986;79:221-5.

13 Alstead EM, Nelson-Piercy C. Inflammatory bowel disease in pregnancy. Gut 2003;52:159-61.

14 Bush MC, Patel S, Lapinski RH, et al. Perinatal outcomes in inflammatory bowel disease. J Matern Fetal Neonatal Med 2004;15:237-41.

15 Calderwood AH, Kane SV. IBD and Pregnancy. MedGenMed 2004;6:14.

16 Cornish J, Tan E, Teare J, et al. A meta-analysis on the influence of inflammatory bowel disease on pregnancy. Gut 2007;56:830-7.

17 Van der Woude CJ, Kolacek S, Dotan I, et al. European evidenced-based consensus on reproduction in inflammatory bowel disease. J Crohns Colitis 2010;4:493-510.

18 Christensen LA, Rasmussen SN, Hansen SH. Disposition of 5 -aminosalicylic acid and $\mathrm{N}$-acetyl-5-aminosalicylic acid in fetal and maternal body fluids during treatment with different 5 -aminosalicylic acid preparations. Acta Obstet Gynecol Scand 1994;73:399-402.

19 Rahimi R, Nikfar S, Rezaie A, et al. Pregnancy outcome in women with inflammatory bowel disease following exposure to 5 -aminosalicylic acid drugs: a meta-analysis. Reprod Toxicol 2008;25:271-5.

20 Colombel JF, Brabant G, Gubler MC, et al. Renal insufficiency in infant: side-effect of prenatal exposure to mesalazine? Lancet 1994;344:620-1.

21 Mogadam M, Dobbins WO, Korelitz BI, et al. Pregnancy in inflammatory bowel disease: effect of sulfasalazine and corticosteroids on fetal outcome. Gastroenterology 1981;80:72-6.

22 Casanova MJ, Chaparro M, Domènech E, et al. Safety of thiopurines and anti-TNF- $\alpha$ drugs during pregnancy in patients with inflammatory bowel disease. Am J Gastroenterol 2013;108:433-40.

23 Akbari M, Shah S, Velayos FS, et al. Systematic review and meta-analysis on the effects of thiopurines on birth outcomes from female and male patients with inflammatory bowel disease. Inflamm Bowel Dis 2013;19:15-22.

24 Coelho J, Beaugerie L, Colombel JF, et al. Pregnancy outcome in patients with inflammatory bowel disease treated with thiopurines: cohort from the CESAME Study. Gut 2011;60:198-203.

25 Jharap B, de Boer NKH, Stokkers P, et al. Intrauterine exposure and pharmacology of conventional thiopurine 
therapy in pregnant patients with inflammatory bowel disease. Gut 2014;63:451-7.

26 Teruel C, López-San Román A, Bermejo F, et al. Outcomes of pregnancies fathered by inflammatory bowel disease patients exposed to thiopurines. Am J Gastroenterol 2010;105:2003-8.

27 Feagins LA, Kane SV. Sexual and reproductive issues for men with inflammatory bowel disease. Am J Gastroenterol 2009;104:768-73.

28 Francella A, Dyan A, Bodian C, et al. The safety of 6-mercaptopurine for childbearing patients with inflammatory bowel disease: A retrospective cohort study. J Gastroenterol 2003;124:9-17.

29 Perales-Puchalt A, Vila Vives JM, López Montes J, et al. Pregnancy outcomes after kidney transplantation-immunosuppressive therapy comparison. J Matern Fetal Neonatal Med 2012;25:1363-6.

30 Bar Oz B, Hackman R, Einarson T, et al. Pregnancy outcome after cyclosporine therapy during pregnancy: a meta-analysis. Transplantation 2001;71:1051-5.

31 Hoeltzenbein M, Stieler K, Panse M, et al. Allopurinol Use during Pregnancy-Outcome of 31 Prospectively Ascertained Cases and a Phenotype Possibly Indicative for Teratogenicity. PLoS One 2013;8:e66637.

32 Miller SL, Wallace EM, Walker DW. Antioxidant therapies: a potential role in perinatal medicine. Neuroendocrinology 2012;96:13-23.

33 Schnitzler F, Fidder H, Ferrante M, et al. Outcome of pregnancy in women with inflammatory bowel disease treated with antitumor necrosis factor therapy. Inflamm Bowel Dis 2011;17:1846-54.

34 Bortlik M, Machkova N, Duricova D, et al. Pregnancy and newborn outcome of mothers with inflammatory bowel diseases exposed to anti-TNF- $\alpha$ therapy during pregnancy: three-center study. Scand J Gastroenterol 2013;48:951-8.
35 PIANO: A 1000 Patient Prospective Registry of Pregnancy Outcomes in Women With IBD Exposed to Immunomodulators and Biologic Therapy. http://download.journals.elsevierhealth. com/pdfs/journals/0016-5085/PIIS0016508512605617.pdf

36 Heller MM, Wu JJ, Murase JE. Fatal case of disseminated BCG infection after vaccination of an infant with in utero exposure to infliximab. J Am Acad Dermatol 2011;65:870.

37 Bar-Oz B, Moretti ME, Boskovic R, et al. The safety of quinolones--a meta-analysis of pregnancy outcomes. Eur J Obstet Gynecol Reprod Biol 2009;143:75-8.

38 Cappell MS. Risks versus benefits of gastrointestinal endoscopy during pregnancy. Nat Rev Gastroenterol Hepatol 2011;8:610-34.

39 Bulas D, Egloff A. Benefits and risks of MRI in pregnancy. Semin Perinatol 2013;37:301-4.

40 Moffatt DC, Ilnyckyj A, Bernstein CN. A population-based study of breastfeeding in inflammatory bowel disease: initiation, duration, and effect on disease in the postpartum period. Am J Gastroenterol 2009;104:2517-23.

41 Klement E, Cohen RV, Boxman J, et al. Breastfeeding and risk of inflammatory bowel disease: a systematic review with meta-analysis. Am J Clin Nutr 2004;80:1342-52.

42 Klotz U, Harings-Kaim A. Negligible excretion of 5-aminosalicylic acid in breast milk. Lancet 1993;342:618-19.

43 Silverman DA, Ford J, Shaw I, et al. Is mesalazine really safe for use in breastfeeding mothers? Gut 2005;54:170-1.

44 Nielsen $\mathrm{OH}$, Maxwell C, Hendel J. IBD medications during pregnancy and lactation. Nat Rev Gastroenterol Hepatol 2014;11:116-27.

45 Mahadevan U, Cucchiara S, Hyams JS, et al. The London Position Statement of the World Congress of Gastroenterology on Biological Therapy for IBD with the European Crohn's and Colitis Organisation: pregnancy and pediatrics. Am J Gastroenterol 2011;106:214-23. 